\title{
Finite Element Simulation of Magnesium Alloy AZ31 Tube Bending
}

\author{
Wenyun $\mathrm{WU}^{1, \mathrm{a}^{*}}, \mathrm{Lv} \mathrm{XIAO}^{2, \mathrm{~b}}$ and Donghong WANG ${ }^{1, \mathrm{c}}$ \\ ${ }^{1}$ School of Materials Engineering, Shanghai University of Engineering Science, Shanghai,201620, \\ China \\ ${ }^{2}$ Shanghai Aerospace Precision Machinery Research Institute, Shanghai 201600, China \\ a*Wenyjlu@126.com, balno_2001_0@163.com, ${ }^{\text {c} w d h c e t 6 @ 163 . c o m ~}$
}

\begin{abstract}
A finite element method based model has been developed for magnesium alloy AZ31 tube bending process simulation, using the tensile (for bend outer radius) and compressive (for bend inner radius) properties of the AZ31 alloy at the bending temperature of $150^{\circ} \mathrm{C}$. The results shown that very high compressive stresses are developed in the bend inner radius, limiting the minimum bend radius for the AZ31 tube. The simulation results suggest the minimum centerline bend radius to the tube outer diameter ratio is 1.5 to 2 . The maximum diameter to thickness ratio is 30 .
\end{abstract}

\section{Introduction}

Magnesium alloy extrusions, especially hollow sections, offer attractive mass saving opportunities for automotive light weighting with potential applications in instrument panel beams, seat frames, roof frames, bumper beams, radiator supports, engine cradles and subframes [1]. Bending is often required to form automotive tubular components. The bendability of magnesium alloy tubes is different compared to aluminum and steel tubes due to different mechanical properties and formability of the materials [2,3]. There are many factors, including temperature, die design and tube surface conditions, influencing the bending deformation and process control [4]. The effects of bending process parameters and tube initial microstructure on the bendability of magnesium alloy extrusions were studied in a previous report [5].

Numerical simulation of metal forming has become a routine practice, which has greatly assisted process and tooling development for many forming processes such as hydroforming. Simulation tools using finite element method (FEM) have been developed for bending of steel and aluminum alloy tubes. These tools can accurately represent the tool and die geometries, boundary constrains, surface interactions, and provide useful assistance in selecting proper tube materials and developing efficient bending process parameters. Magnesium alloys, with plastic anisotropy due to their hexagonal close packed (hcp) crystal structure, present significant challenges in bending process simulation.

The aim of this paper is to develop a simulation procedure for AZ31 tube bending

* Corresponding author:Wenyjlu@126.com 
process. The stress and strain distributions in tube bending were analyzed for understanding of bending deformation mechanisms. The ultimate geometrical parameters of bending process were analyzed.

\section{Modeling and Experimental Procedures}

A representative finite element model is established using the ANSYS code. Eight-node hexahedron solid elements were selected to represent the tube wall. The deformation of the element is three-dimensional and its shape is quadrilateral. The elements have three rotational degrees of freedom, and three displacement degrees of freedom. In order to reduce the computer-processing time during FEM calculations, only half of the tube in the circumferential direction is required to be modeled. There are essentially two different boundary conditions in the tube bending experiment setup which need to be considered in the model. The first boundary condition is "glue" (no relative movement), which includes the interface between the tube and the clamp die, and the one between the tube and the straight part of the bend die. The second boundary condition is "friction", which applies to the interface between the tube and other parts of dies where Coulomb-type friction is defined. The tube material is defined as elastic-plastic type. The plasticity properties of material are defined with tables based on test curves (Fig. $1 \mathrm{a}, \mathrm{b}$ ). Two material models are defined. The inner radius of the tube is described by the compressive stress-strain curve, while the tube outer radius is defined by the tensile curve (Fig. $1 \mathrm{c}$ ).
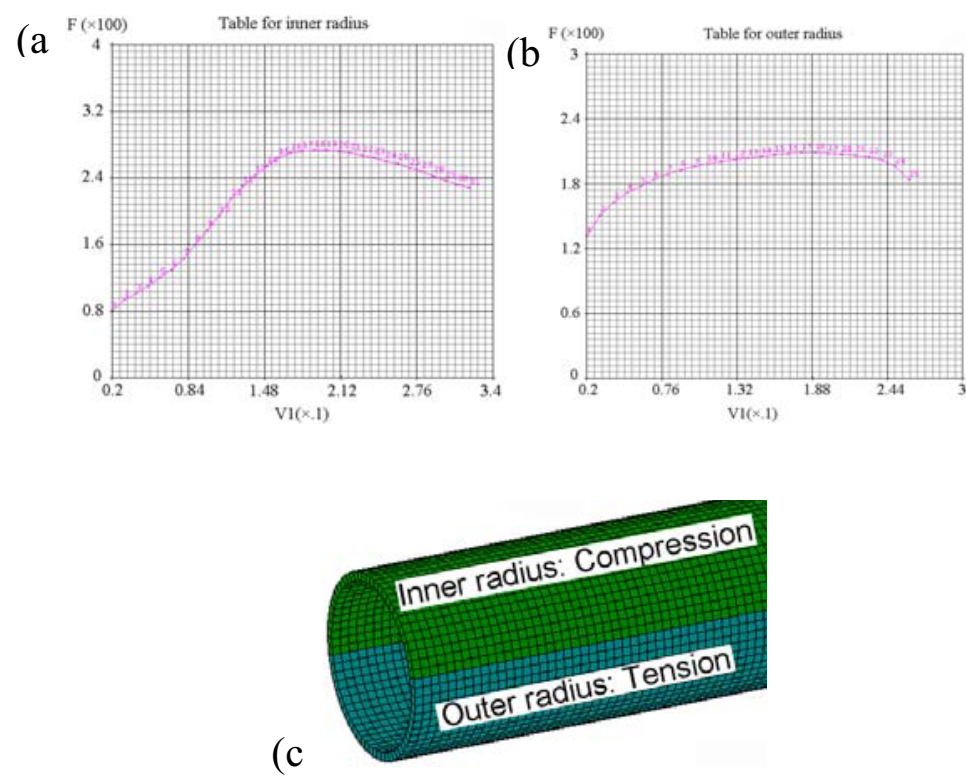

Fig. 1 Stress-strain curves of AZ31 tube at $150^{\circ} \mathrm{C}(\mathrm{a}, \mathrm{b})$ input to the bending model (c)

The modulus of elasticity for the material model is obtained from the initial linear region of the test stress-strain curves, and a value of 0.35 is used as the Poisson's ratio. The loading procedure includes three steps, i.e. bending, releasing and mandrel withdrawal. Fig. 2 shows the final model and the bending process. 

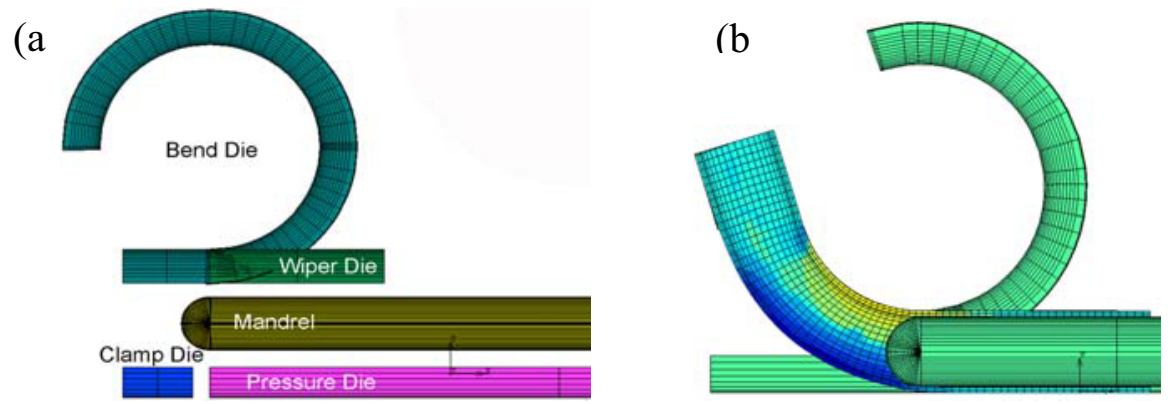

Fig. 2 Finite element model for tube bending simulation: (a)dies setup, (b)bending process

\section{Results}

\subsection{Stress and Strain Distributions}

Fig. 3 shows the maximum equivalent stress calculation along the tube inner and outer radii at different bending angles. The stress level at the bend inner radius is lower than that of outer radius at low bending angles. The stresses in both sides increase rapidly with bending angle and the stress at the inner radius exceeds that of the outer radius before both reach a stable state at about $30^{\circ}$ angle. This condition agrees well with the assumption tension in outer radius and compression in inner radius of the tube as shown in Fig. 1. Fig. 3 also shows that the stable stress level (in compression) in the bend inner radius is considerably higher than that of outer radius (in tension).
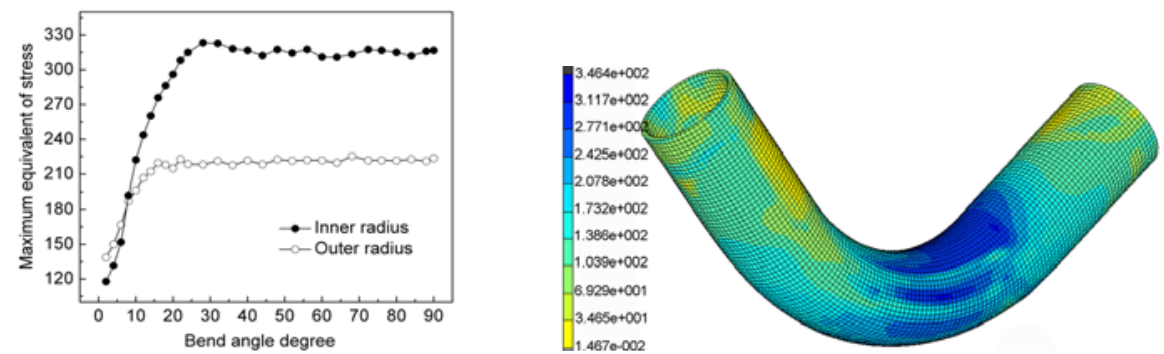

Fig. 3 Maximum equivalent stress at inner and outer radii of tube ( $2 \mathrm{D} / 90^{\circ}$ bend $)$

Fig. 4 shows the maximum equivalent plastic strain along the tube inner and outer radii. The plastic strain in the inner radius is a slightly higher than that of outer radius at low bend angles up to about $15^{\circ}$. Then, the strains in both sides gradually increase with the bend angle, with the inner strains slightly higher than the outer strain for bend angles greater than about $25^{\circ}$. 

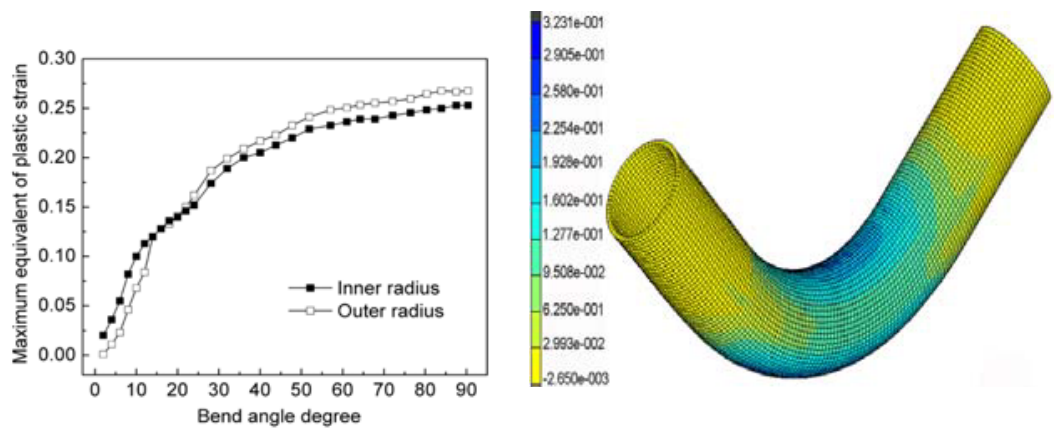

Fig. 4 Maximum equivalent strain at inner and outer radii of tube $\left(2 \mathrm{D} / 90^{\circ}\right.$ bend $)$

\subsection{Effects of Relative Bend Radius}

Fig. 5 shows the effects of relative bend radius on the bendability. It is evident from Fig. 5 (a) and (b) that the maximum wall thinning and cross-section ovality increase for tighter bends (smaller relative bend radii). On the contrary, the spring-back decreases with the reduction of relative bend radius as shown in Fig. 5 (c). Excessive deformation and cracks can be seen at 1D bend (Fig. 5 (d)). Therefore, a minimum bend radius of 2D is recommended in design and manufacturing of AZ31 magnesium alloy tubes.

\subsection{Effects of Tube Diameter / Thickness Ratio}

Fig. 6 shows the effects of the relative wall thickness (diameter / thickness ratio) on the bendability of the AZ31 alloy tubes. As shown in Fig. 6 (a), the wall thinning increases slightly with the diameter / thickness ratio up to 20, and then decreases. The cross-section ovality increases directly with relative wall thickness as shown in Fig. 6 (b). On the other hand, the spring-back has essentially no relationship with the diameter / thickness ratio, as shown in Fig. 6 (c.). Fig. 6 (d) shows the bending simulation results when the diameter / thickness ratio is 40 . It can be seen that the tube outer radius severely collapses along the bend radius direction.
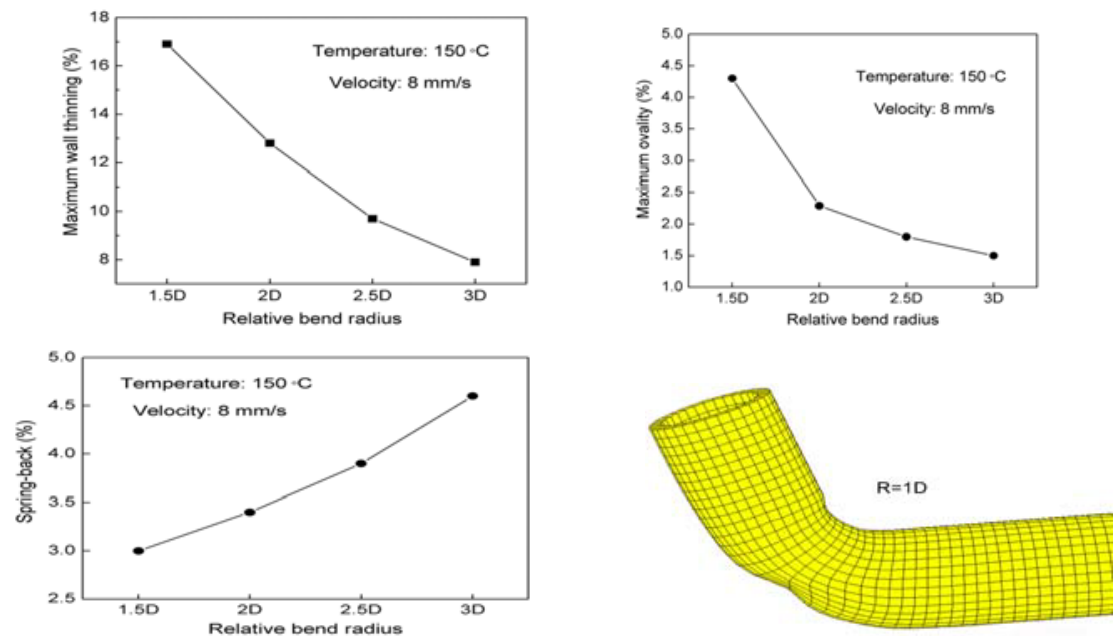

Fig. 5 Effects of relative bend radius on the bendability 

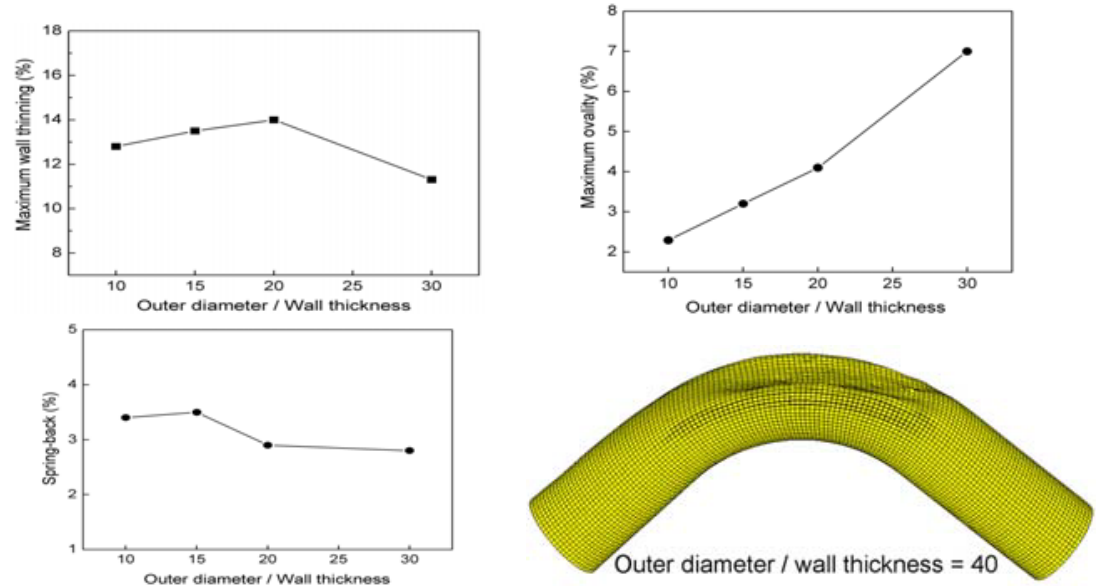

Fig. 6 Effects diameter/thickness ratio on the bendability $\left(2 \mathrm{D} / 90^{\circ}\right.$ bend $)$

\section{Discussion}

The bending operation is a plastic deformation process. At early stages of bending, low stress but higher plastic strain is developed in the bend inner radius compared to those in outer radius. This is due to the lower compressive yield strength in compression than tension, leading to larger deformation at lower stress in the compression condition. With the increasing bend angle, slightly larger plastic strains but significantly lower stress are developed in the outer radius compared with the inner radius, due to the considerably higher strain-hardening rate observed in the compression. It is noted that very high compressive stresses are calculated in the inner radius, higher than the compressive strength at bending temperature $150^{\circ}$.

While the tube outer diameter is often determined by the structural requirements, the tube wall thickness and bend radius need to be selected in designing tubular components for mass-efficiency and low-cost manufacturing. The simulation results suggest a minimum bend radius of $1.5 \mathrm{D}$ or $2 \mathrm{D}$, with the $1.5 \mathrm{D}$ bend producing more thinning and ovality but less spring-back. Small bend radii result to higher plastic strains, and leading to higher cross-section ovality. For a smaller bend radius, the total volume of the bent tube is smaller; hence, less elastic strain energy stored and less spring-back in the part. While $2 \mathrm{D}$ is generally recommended as the minimum bend radius.

Thin-wall tubes show more radial strain. The ovality, rather than the thinning or spring-back, is showing more sensitivity to the wall thickness. Additionally, the flattening of a thinner-wall tube on the outer bend radius can reduce the tangent plastic strain, leading to less wall thinning. The results suggest the maximum diameter/thickness ratio is 30 .

\section{Conclusions}

FEM simulation of the AZ31 bent tubes were studied. High compressive stresses are calculated in the bend inner radius. $2 \mathrm{D}$ is generally recommended as the minimum bend radius. The maximum diameter / thickness ratio is 30 . When the ratio exceeds 30 , the tube is showing excessive flattening or cracking. 


\section{Acknowledgements}

This research was supported by the Startup Project of Shanghai University of Engineering Science (No. 2014-32) and the Young Teachers Developing Plan of Shanghai Municipal Education Commission (No. ZZgcd14012).

\section{References}

1. Luo, A.A. Wrought Magnesium Alloys and Manufacturing Processes for Automotive Applications. Transactions - Journal of Materials and Manufacturing. SAE, Warrendale, PA, U.S.A. (2005) 411-421.

2. Kohzu, M., Yoshida, F., Higashi, K. Evaluation of Press Formability in Magnesium Alloy. Mater. Sci. Forum (2003) 321-326.

3. Beer, A.G., Barnett, M.R. The Hot Working Flow Stress and Microstructure in Magnesium AZ31. Magnesium Technol. (2002) 193-198.

4. Houliara, S., Karamanos, S.A. Buckling and Post-Buckling of Long Pressurized Elastic Thin-Walled Tubes under In-Plane Bending. International Journal of Non-Linear Mechanics 41, (2006) 491-511.

5. Wu, W.Y., Zhang, P., Zeng, X.Q., Jin, L., Yao, S.S., Luo, A.A. Bendability of the Wrought Magnesium Alloy AM30 Tubes Using a Rotary Draw Bender. Mater. Sci. Eng. A, 486, (2008) 596-601. 